No. 1-2

Abstracts. 43. Österreichischer HNO-Kongress 1999

Rust, Österreich, 15.-19. September 1999

Primarius Dr. med. univ. Robert Pavelka, Wiener Neustadt, Österreich

No. 3-4

Festschrift

zum 75. Geburtstag von Prof. Dr. med. Karl-Heinz Vosteen

\section{Vorwort zur Festausgabe}

81 Der Einfluss der Stapesfussplattenfläche auf den Druck in der Perilymphe und die Auslenkung der Basilarmembran Influence of the Area of Stapes Footplate Prostheses on Perilymphatic Pressure and Basilar Membrane Displacement L'influence de la surface de la platine sur la pression dans la périlymphe et sur le déplacement de la membrane basilaire Arnold, W.; Böhnke, F.; Scherer, E. (München)

87 Untersuchungen zu einer möglichen Masernviruspersistenz in der Otosklerose

Investigations of a Possible Measles Virus Persistence in Otosclerosis

Recherche sur la persistence possible du virus de la rougeole associée à l'otospongiose

Niedermeyer, H.P.; Arnold, W.; Schuster, M.; Wesemeier, K.; Kramer, J.; Baumann, C.; Neubert, W.J. (München)

92 Zur Bedeutung der Melanozyten des Innenohres im Rahmen der Spiralganglienzelldegeneration beim Akustikusneurinom The Role of the Melanocytes of the Inner Ear in Spiral Ganglion Cell Degeneration of Acoustic Neuroma Signification de la présence de mélanocytes en cas de dégénérescence du ganglion spiral consécutive à un neurinome de l'acoustique

Sánchez-Hanke, M.; Koch, U.; Ahrendt, I.; Ußmüller, J. (Hamburg)

98 Der Einfluss von Glukokortikoiden bei der Therapie des Hörsturzes

Influence of Glucocorticoids in the Treatment of

Sudden Sensorineural Hearing Loss (SNHL)

L'influence des glucocorticoïdes dans le traitement de la perte brutale de l'audition

Alexiou, C.; Arnold, W.; Fauser, C.; Gloddek, B.; Fuhrmann, S.; Lamm, K. (München)

105 Results of Pediatric Cochlear Implantation Compared with Results Obtained with Hearing Aids

Ergebnisse der Cochlea-Implantation bei Kindern im Vergleich zu Ergebnissen mit Hörgeräten

Résultats de l'implantation cochléaire pédiatrique en comparaison avec les résultats obtenus avec appareil auditif Kiefer, J.; Pfennigdorff, T.; Spelsberg, A.; Gall, V. (Frankfurt); Diller, G. (Friedberg); von Ilberg, C. ( Frankfurt)
115 Zentral-auditive Verarbeitungsstörungen bei Lese- und Rechtschreibschwäche - Ergebnisse einer retrospektiven Erhebung Central Auditory Processing Disorder and Dyslexia Clinical Data of 53 Children

Troubles auditifs centraux et dyslexie: données chez 53 enfants Matulat, P.; Riebandt, M.; Lamprecht-Dinnesen, A. (Münster)

120 Langzeitergebnisse von Patienten mit einem Cochlea-Implantat Long-Term Results of Cochlear Implant Patients Résultats à long terme de patients avec un implant cochléaire Riebandt, M.; Sandrieser, P.; Matulat, P.; Lamprecht-Dinnesen, A.; Seifert, E. (Münster)

129 Mechanismen der Wachstumsregulation bei Karzinomen - ein Überblick

Mechanisms of Growth Regulation in Carcinomas - A Survey Des mécanismes de la régulation de l'augmentation des carcinomes - un résumé

Ganzer, U.; Haas, I.; Bier, H.; Koldovsky, P. (Düsseldorf)

137 Ist die Positronen-Emissions-Tomographie (PET) zum Nachweis tumorbefallener Halslymphknoten bei Plattenepithelkarzinomen des Kopf-Halsbereiches in der klinischen Routine sinnvoll einsetzbar?

${ }^{18}$ F-FDG-PET in Lymph Node Detection of Head and Neck Squamous-Cell Carcinomas

Détection de l'envahissement ganglionnaire dans les cancers à cellules squameuses de la tête et du cou par TEP au ${ }^{18}$ F-FDG dans les conditions de la pratique clinique

Kau, R.J. (Krefeld); Alexiou, C.; Werner, M.; Schwaiger, M.; Arnold, W. (München)

144 Die Lokalisation von Mehrfachtumoren in den Luft- und oberen Speisewegen als Hinweis auf eine diagonale Rauchstrasse Localisation of Multiple Tumours in the Aero-Digestive Tracts as an Indication for a Diagonal Route of Smoke Inhalation La localisation de tumeurs multiples au niveau du tractus aérodigestif en tant que signe de l'existence d'une route diagonale de l'inhalation de la fumée Lamprecht, J. (Essen); Löser, S. (Aachen)

149 Zur chirurgischen Therapie der Amiodaron (Cordarex)induzierten Hyperthyreose und thyreotoxischen Krise Surgical Treatment of Amiodarone-Induced Thyrotoxicosis Traitement chirurgical de l'hyperthyréose due à l'amiodarone Biermann, E.; Morgenstern, C. (Hamburg)

155 Effect of Diode Laser on Mucosal Swelling of Outpatients with Perennial Allergic Rhinitis. A Preliminary Report Die Wirkung einer High-Power-Dioden-Laserbehandlung der Schleimhaut der unteren Nasenmuschel bei ambulanten Patienten mit perennialer allergischer Rhinitis Effet du laser diode sur la tuméfaction muqueuse de la rhinite allergique perannuelle chez les patients traités ambulatoirement Nishimoto, J.; Kumazawa, H.; Kumazawa, T.; Yamashita, T. (Osaka)

160 Bilaterale chronische Parotitis bei unerkanntem MünchhausenSyndrom. Ein nicht ganz gewöhnlicher Fall Bilateral Chronic Parotitis by Unrecognized Munchhausen's Syndrome

Parotide chronique bilatérale comme manifestation d'un syndrome de Münchhausen

Biermann, E.; Schultz, K.-D.; Morgenstern, C. (Hamburg)

\section{KARGER}

Fax + 41613061234 E-Mail karger@karger.ch www.karger.com 
No. 5

\section{Sudden Deafness}

A Controversial Issue

Guest Editor: Jean-Philippe Guyot, Geneva, Switzerland

Editorial

169 Sudden Deafness: The Missing Questions and Data

Guyot, J.-P. (Geneva)

171 Idiopathic Sudden Sensorineural Hearing Loss.

Reasons for Defeat, Conditions for Victory

Idiopathischer plötzlicher Hörsturz: Gründe des Misserfolgs,

Bedingungen für den Erfolg

Surdité brusque idiopathique: les raisons d'une défaite,

les conditions d'une victoire

Tran Ba Huy, P. (Paris)

178 Sudden Deafness - A Critical Evaluation of Pathogenesis and 'Cure'

Hörsturz: eine kritische Beurteilung der Pathogenese und "Heilung"

Surdité brusque: évaluation clinique des hypothèses pathogéniques et des modalités thérapeutiques Hultcrantz, E. (Linköping)

190 Sudden Deafness and Viral Infections

Hörsturz und Virusinfektionen

Surdité brusque et infection virale

Pitkäranta, A.; Vasama, J.-P.; Julkunen, I. (Helsinki)

198 Sudden Deafness. Reviews by the Research Group of the Japanese Ministry of Health and Welfare

Hörsturz

Surdité brusque

Kanzaki, J. (Tokyo)

203 How Useful Is Corticosteroid Treatment in Cochlear Disorders? Wie nützlich ist eine Therapie mit Kortikosteroiden bei Innenohrerkrankungen?

Quelle est I'utilité du traitement par corticostéroïdes dans les affections cochléaires?

Lamm, K.; Arnold, W. (Munich)

217 Management of Idiopathic Sudden Sensorineural Hearing Loss Behandlung der idiopathischen plötzlichen Perzeptionsschwerhörigkeit

Prise en charge des surdités brusques

Mosnier, I.; Bouccara, D.; Sterkers, O. (Clichy)

224 Congress Calendar • Calendrier des congrès • Kongresskalender
No. 6

Original Papers $•$ Travaux originaux $\bullet$ Originalarbeiten

225 Change of Vestibular Function after Cochlear Implantation Änderung der vestibulären Funktion nach kochleärer Implantation

Changement de la fonction vestibulaire après implantation cochléaire

Szirmai, Á.; Ribári, O.; Répássy, G. (Budapest)

229 Metal Ganglionitis: Clinical Pathologic Correlation in Idiopathic Facial Paralysis (Bell's Palsy)

Meatale Ganglionitis: klinisch-pathologischer Zusammenhang mit der idiopathischen Fazialisparese (Bell-Lähmung)

Ganglion méatal: corrélation entre clinique et pathologie dans la paralysie faciale idiopathique (paralysie de Bell) Gacek, R.R.; Gacek, M.R. (Mobile, Ala.)

239 Halsdissektion: Konzept zur histopathologischen Aufarbeitung Neck Dissection: Concept for Histopathological Exploration Evidement cervical - Concept pour l'examen histopathologique Danninger, R.; Humer, U.; Beham, A.; Jakse, R. (Graz)

245 Les cellulites cervicales diffuses. A propos de 5 observations Zervikale Phlegmone Diffuse Cervical Cellulitis Vigne, P.; Duvillard, C.; Romanet, P. (Dijon)

252 Stapedotomy Piston Diameter - Is Bigger Better? Pistondurchmesser für Stapedotomie - je grösser desto besser? Diamètre du piston de stapédotomie: un diamètre élevé est-il préférable?

Teig, E.; Lindeman, H.H. (Oslo)

Leitlinien der Deutschen Gesellschaft für Hals-Nasen-OhrenHeilkunde, Kopf- und Halschirurgie

258 Antibiotikatherapie der Infektionen an Kopf und Hals (Konsensusbericht)

Im Auftrag des Präsidiums herausgegeben von Federspil, P. (Homburg/Saar)

270 Onkologie des Kopf-Hals-Bereiches (Konsensusbericht) Im Auftrag des Präsidiums herausgegeben von Bootz, F. (Leipzig)

288 Congress Calendar • Calendrier des congrès • Kongresskalender

289 Author Index Vol. 9, 1999

290 Subject Index Vol. 9, 1999 